\title{
Research and Application of Infrared Leak Detection Technology for SF6 Equipment
}

\author{
Hai Tianshu, Li Bo, Wu Yaowei, Li Tianci, Song Yuanlin, Su Yang, Hu Yang, Hao Wenfeng, \\ Liu Chenyang, Han Lin
}

Fushun Power Supply Company, Liaoning Electric Power Company Limited, State Grid, China

Keywords: SF6 gas; Infrared imaging; Leak detection technology

\begin{abstract}
The infrared leak detection technology of SF6 equipment can detect and locate the gas leakage without power failure, and realize the real-time transmission of fault image, improve the efficiency of maintenance, ensure the safe and stable operation of SF6 electrical equipment. In this paper, the problem of SF6 gas leakage in Benxi Hedi 220kV substation and Liaoyang Shoushan $66 \mathrm{kV}$ substation is discussed. The infrared leak detection technology of SF6 equipment is studied and analyzed. This paper discusses the harm, advantage, principle and field application of infrared leak detection technology. According to different leakage reasons, the relative solutions are summarized.
\end{abstract}

\section{Introduction}

SF6 was first used as an insulating medium in 1940.With the rapid development of power grid technology, SF6 gas is widely used in various power equipment as a medium with excellent insulation and arc extinguishing performance. Due to the influence of quality, process, service time, operating environment and natural factors, SF6 equipment has gas leakage. The leakage of SF6 gas, especially arc decomposition gas, has adverse effects on the environment, personal safety and the operation of power grid equipment. The leakage of SF6 gas will directly affect the insulation performance and arc extinguishing ability of the restarting equipment, which will lead to the locking of the SF6 circuit breaker and even the breakdown of the internal insulation of the equipment. Therefore, leak detection of SF6 equipment is one of the most important work in operation and maintenance of gas insulation equipment.

The traditional detection mostly uses qualitative SF6 leak detector or quantitative SF6 leak detector. In the process of leak detection, the distance from the leak point is close, and the workload is large. Power outages must be detected in addition to GIS and some equipment of circuit breakers. The limitation of the detection seriously affects the normal operation of the equipment and prolongs the operation and outage time of the equipment. In recent years, infrared imaging technology, as a non-contact live detection technology, has been effectively used in leak detection of SF6 equipment, which is conducive to the safe operation of equipment and the development of condition maintenance.

\section{The necessity of Infrared Leak Detection}

The harm of SF6 gas leakage.

Physical hazards. Pure SF6 gas is a colorless, odorless, toxic and flammable halogen compound. But SF6 is a Greenhouse Effect gas, it is 23900 times more damaging to the ozone layer than carbon dioxide and has a potential hazard of 3, 200 years. The relative density of SF6 gas is $6.16 \mathrm{~g} / \mathrm{cm} 3\left(20^{\circ} \mathrm{C}, 0.1 \mathrm{MPa}\right)$, which is 5 times of that of air. When working indoors, the gas accumulates on the ground once the SF6 inflatable equipment leaks. When the human body inhales a large amount of $80 \%$ SF6 and 20\% O2 mixed gases, it can cause limb numbness and even asphyxiation.

Chemical hazards. The chemical properties of SF6 are very stable, but under the action of high power arc, spark discharge and corona discharge, SF6 gas can decompose and dissociate many 
products. Once the equipment leaks, water vapor enters the device at the leak point. It not only affects the insulation of equipment, but also produces toxic products such as SO2 and H2S, which are produced by chemical reaction with SF6 gas. In the event of a leak, the cause must be identified and eliminated.

\section{Advantages of Infrared Leak Detection Technology}

High detection efficiency. As a live detection method, infrared leak detection technology can be used to directly observe and collect images from the ground under the condition that the equipment is without power. Infrared leak detection technology does not need a specific background, the equipment response speed, high sensitivity, easy to carry. Compared with the traditional leak detection method, it can find leakage point more intuitively and quickly. Using infrared leak detection technology to detect leakage of SF6 equipment regularly, SF6 leakage defects can be found before low voltage alarm. As a result, the blackout time and maintenance workload are greatly reduced, and the direct, reliable and clear decision basis is provided for the Power outage treatment. After power outages treatment can be carried out site review, to avoid secondary power outages.

Economic security. The infrared leak detection technology is suitable for monitoring the equipment which is difficult to reach by the contact measuring tool, and can detect the potentially dangerous leakage from a few meters away.SF6 gas is expensive, about 200000 yuan per ton, the annual loss caused by gas leakage is enormous. Infrared leak detection technology can locate and deal with leakage points in time and effectively, and reduce gas leakage. Infrared leak detection technology is less affected by the external environment. It can be carried out in light rain, wind power below level 4, and narrow space. This will avoid the detection of environmental factors affecting the safe operation of equipment, resulting in economic losses.

Environmental protection. Infrared leak detection technique is intuitive and accurate, it can detect and detect SF6 gas leakage defects in a timely and effective manner. In this way, the gas leakage defects of the gas insulation equipment are prevented from being discovered when the gas pressure alarm is developed, reducing the leakage of SF6 gas, thus reducing the atmospheric damage caused by greenhouse effect.

\section{Principle of Infrared Leak Detection Technology}

All objects in nature, when their temperature is greater than the absolute temperature $\left(-273^{\circ} \mathrm{C}\right)$, there is an irregular movement of molecules and atoms, their surfaces are constantly radiating infrared light. Infrared leak detection technology makes use of the difference of infrared absorption characteristics between SF6 gas and air to form different infrared images. It allows unseen gas leakage to occur with the support of advanced ultra-high sensitivity infrared detector and infrared detection techniques.

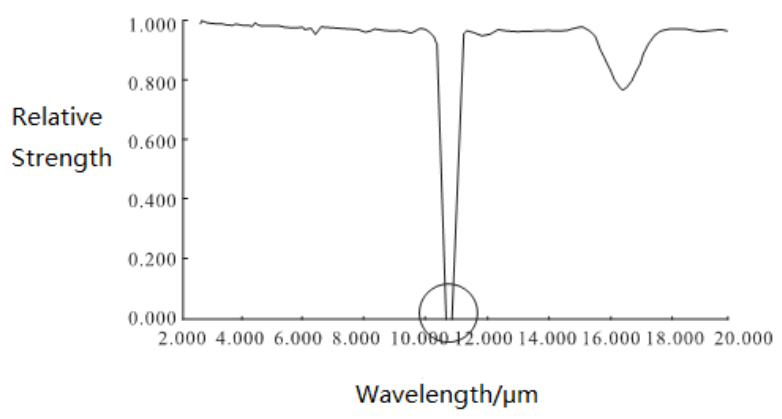

Fig. 1 SF6 gas infrared absorption spectrum

The strongest absorption of SF6 gas is in the range of $10.6 \mu \mathrm{m}$, and its infrared transmittance is 
poor in this band. Therefore, by limiting the working band of the infrared imager to $10.3 \mu \mathrm{m}$ or $10.7 \mu \mathrm{m}$, the SF6 leakage gas which cannot be seen by the naked eye can form a soot like dynamic image on the instrument screen. The infrared absorption spectrum of SF6 gas is shown in Fig. 1 and the imaging schematic diagram is shown in Fig. 2.

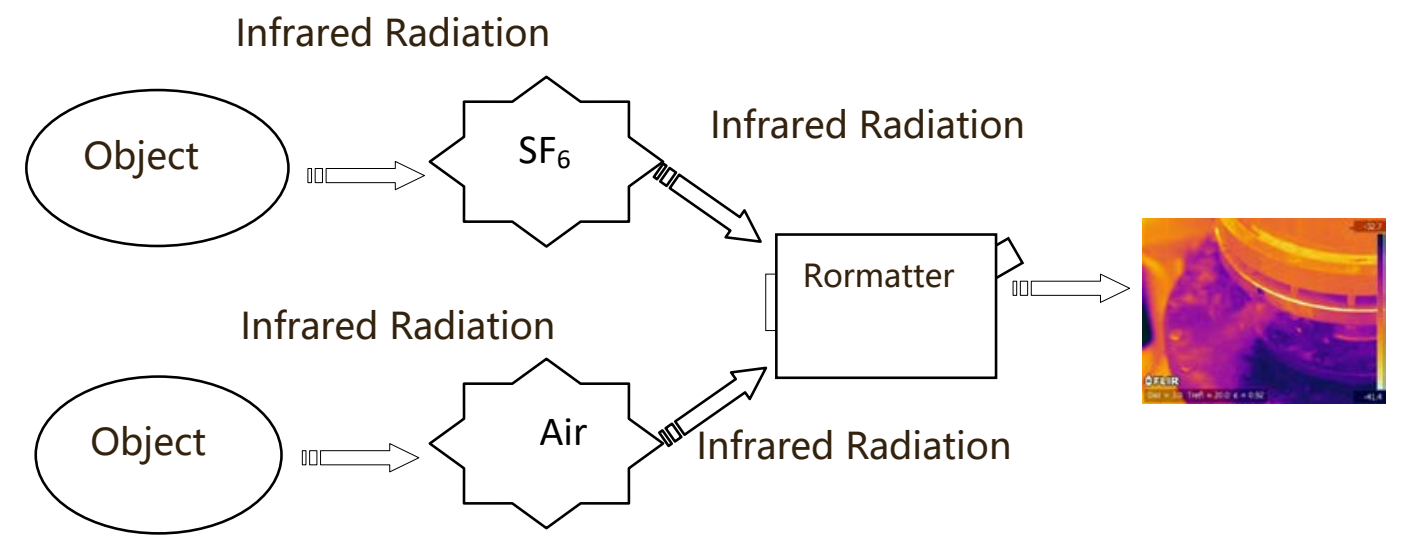

Fig. 2 Working principle of infrared imager

\section{Field Application of Infrared Leak Detection Technology}

Leak detection

Equipment leakage is not a single continuous, most of the sudden or intermittent possibility, usually a device leak detection test takes 1-2 hours. The same observation point where there may be leakage should be concluded by the two experimenters taking turns from different observation points. In general, the duration of each observation should last more than 1 minute.SF6 equipment may have multiple leak points, each bolt, every flange may have leak points, in the leak detection process must be patient, careful observation.

The work site uses the GF300 infrared thermal imager produced by FLIR systems Company of the United States. From December 2015 to November 2017, several 66 kV, 220 kV and 500kV substations in Liaoning Province were tested by infrared detection. Taking Benxi Hedi 220kV substation and Liaoyang Shoushan $66 \mathrm{kV}$ substation as examples, the application of infrared leak detection technology is introduced.

Because the radiation ability of SF6 gas is stronger than that of air at $1010 \sim 11 \mu \mathrm{m}$ band, SF6 gas can be seen fluttering in the air clearly near the leakage point by infrared leak detector.Fig.3, Fig.4 is a typical illustration of infrared leak detection of transformer oil and gas bushing in Benxi Hedi 220kV substation on January 3, 2015. Fig.5, Fig.6 is a typical illustration of infrared leak detection of HGIS equipment in Liaoyang Shoushan 66kV substation on December 26,2016.

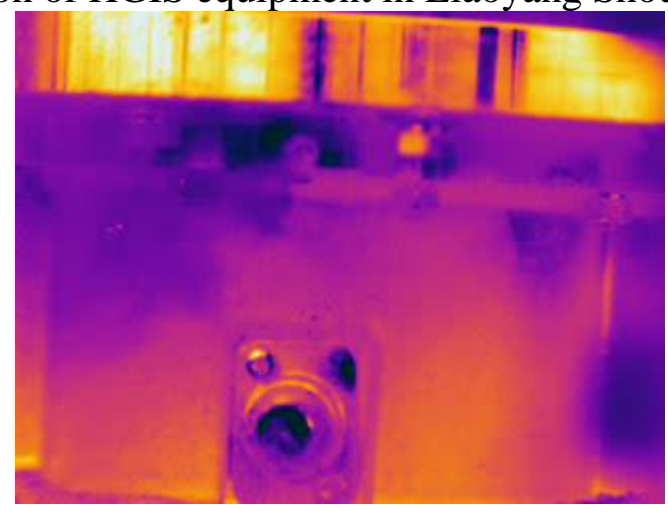

Fig.3

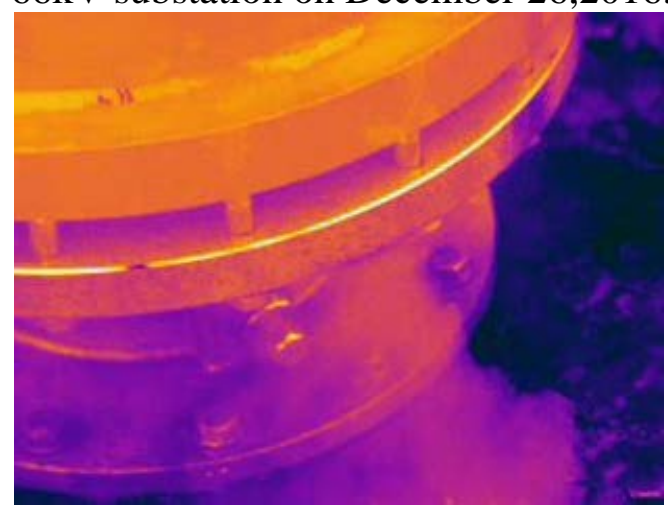

Fig.4

Ransformer oil and gas bushing in Benxi Hedi 220kV substation 


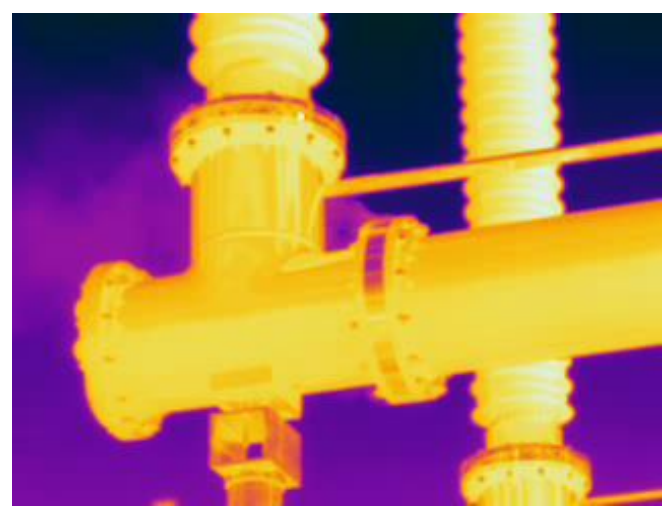

Fig.5

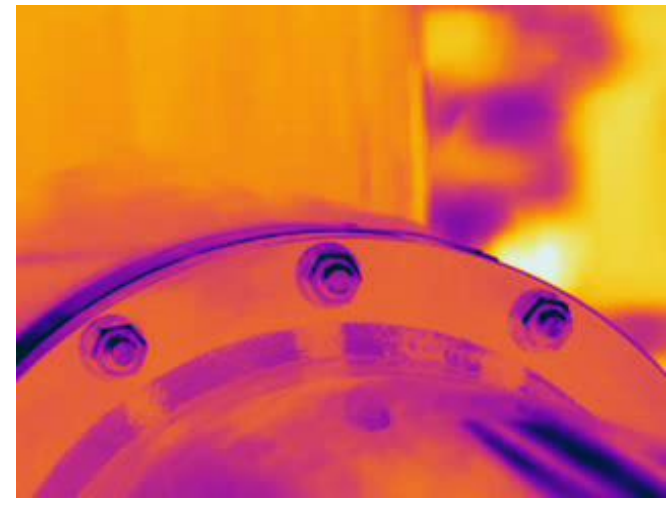

Fig.6

HGIS equipment in Liaoyang Shoushan 66kV substation

\section{Result analysis and processing method}

As far as the field test results of Benxi Hedi $220 \mathrm{kV}$ substation and Liaoyang Shoushan 66kV substation are concerned, Gas leakage occurs in dynamic seal, pipeline aging or flange sealing ring aging, other equipment defects such as trachoma are rare.

There are several main reasons for the leakage: Process problems during vacuum casting, cooling or curing of basin insulators lead to quality defects; The waterproof sealant between flange and basin insulator is not good. The equipment has been running for a long time after sudden change of climate and alternate rain and snow, under the action of gas expansion force, sealing surface deformation, crack, link bolt pre-tightening force failure and so on lead to air leakage.

Judge the degree of defect according to the gas supply record, the family defect of the equipment, and the experience. Strengthen inspection of defect sites and check regularly. An infrared leak detector should be used after temperature change or heavy rain and snow weather, and pay close attention to the pressure gauge value changes. Paint the waterproof glue on unsealed parts strictly. Fasten bolts according to the corresponding process requirements and replace the serious parts where cracks occur.

\section{Conclusions}

Compared with conventional methods, infrared leak detection technology can be used for non-contact and long-distance detection without power failure, and no specific detection background is required. The infrared leak detection technology has high detection sensitivity and can detect and locate leak points directly and quickly, which provides a direct and reliable decision basis for power outage treatment. Infrared leak detection technology is used in installation, operation inspection, outage maintenance and fault tracking, which can effectively reduce the workload of maintenance personnel and the loss caused by outage operation, and ensure the safe and stable operation of SF6 electrical equipment.

\section{References}

[1] Gao Shuguo, Zheng Aiquan, Geng Jianghai. Detection of leakage of SF6 electrical equipment by infrared imaging technology [J]. High-voltage Electrical Apparatus .2010 46(3): 103-105.

[2] Hu Weitao, Sui Shaochen, Han Jianbo. Application of Infrared leak detector in condition maintenance of SF6 Electrical equipment [J]. High-voltage Electrical Apparatus. 2010,46 (10): 90-96.

[3] Zhou Yongqiang, Liu Xiaodong, Zhou Kaifeng. Cause Analysis and treatment measures of 220kV GIS Air leakage defect[J]. Hebei Electric Power Technology.2011,30(4):34-36.

[4] Liu Yanchao. Application of Infrared Imaging Technology in Power system [J]. Science and 
Technology Innovation report 2008(28):10-11.

[5] Yang Yue, che Chuanqiang, Liu Tiecheng. Application of Infrared Imaging Technology in Live leak Detection of SF6 equipment [J]. Inner Mongolia Electric Power Technology. 2012.30(3): 80-83 